\title{
EFFECT OF LATTICE STRUCTURE ON THE POSITRON ANNIHILATION WITH INNER SHELL ELECTRONS
}

\author{
M. ALATALO ${ }^{a}$, P. ASOKA-KUMAR ${ }^{a}$, V. J. GHOSH ${ }^{a}$, B. NIELSEN ${ }^{a}$, K. G. LYNN ${ }^{a} *$, \\ A. C. KRUSEMAN ${ }^{b}, A . V_{\text {VAN VEEN }}^{b}$, T. KORHONEN ${ }^{c}$ and M. J. PUSKA ${ }^{c}$ \\ ${ }^{a}$ Department of Physics, Brookhaven National Laboratory, Building 510A, Upton, NY 11973, USA \\ 'Interfaculty Reactor Institute, Delft University of Technology, 2629 JB Delft, The Netherlands \\ ${ }^{c}$ Laboratory of Physics. Helsinki University of Technology, FIN-02150 Espoo, Finland
}

(Received 6 January 1997; accepted 25 March 1997)

\begin{abstract}
The effects of lattice structure on the high momentum part of the Doppler broadening of the positronelectron annihilation radiation was studied for a number of materials. The momentum distribution of the annihilation radiation was mcasured using two Ge detectors to record "in coincidence" the energies of both the upshifted and downshifted gamma rays. First-principles calculations were performed to complement the experimental data. We present results for diamond and graphite, $\alpha$ and $\beta \mathrm{Sn}$, amorphous and crystalline $\mathrm{Si}$, wellannealed and deformed $\mathrm{Cu}$, and $\mathrm{Fe}$, with and without vacancy clusters. The bulk systems provide a good test case for the present approach, since the delocalized positron has no effect on the surrounding lattice. These results show that the two-detector technique, which exhibits enhanced elemental sensitivity, can also be used as a probe of the geometry of the annihilation environment, and hence can be used for identifying defect structures. (C) 1997 Elsevier Science Ltd. All rights reserved.
\end{abstract}

Keywords: A. elements, C. positron annihilation spectroscopy, D. defects

\section{INTRODUCTION}

The measurement of positron lifetimes, the Doppler broadening (DBAR) and two-dimensional angular correlation of the annihilation radiation (2D-ACAR) have traditionally been used for the identification of defects in solids [1]. In order to conserve momentum, the annihilation of the electron-positron pair produces two gamma rays (the much less likely $3 \gamma$-annihilation is omitted in our discussion) whose relative direction differs from $180^{\circ}$ by the angle $\theta=p_{T} / m_{0} c$, where $m_{0}$ is the electron mass and $c$ is the speed of light. $p_{T}$ is the transverse component of the momentum and can be measured using 2D-ACAR. The longitudinal momentum component $p_{L}$ along the direction of the photon emission is related to the Doppler shift $\Delta E$ by

$$
p_{L}=2 \Delta E / c \text {. }
$$

Significant improvements in the Doppler broadening measurement can be achieved by coincidence measurements of the two annihilation quanta [2-7]. The signalto-noise ratio can be improved further by using two $\mathrm{Ge}$ detectors $[3,5]$, leading to better measurements of the higher momentum region where few annihilation events occur and result in a low count rate.

This higher-momentum part of the DBAR spectrum arises from annihilations with the core electrons. These

*Present address: Materials Research Center, Washington State University, Pullman, WA 99164-2711. electrons retain their atomistic character in a solid, and the momentum distribution resulting from their annihilation can be used for identifying the chemical environment of the annihilation site. The screening of the core electrons by the valence electrons, and hence the penetration of the positron wave function into the core and the partial annihilation rates for each core level depend on the lattice structure or the geometry at the annihilation site. Hence, this technique can be used for identifying both the elemental character as well as the geometry. The propensity of positrons to trap at open-volume defects prior to annihilation thus makes them extremely useful for identifying defects like vacancy-impurity complexes in solids [7].

The positron annihilation characteristics depend on the amount of overlap between the positron and electron wave functions [1]. An increase in the overlap of the positron and electron wave functions results in an increase in both the partial (and hence total) annihilation rates as well as the amplitude of the momentum distribution. Variations in the positron lifetime, which is inversely proportional to the total annihilation, are largely dominated by the contributions of the valence electron-positron annihilation rate, whereas the momentum distribution is equally sensitive to both the valence and core electron contributions. The valence electronpositron overlap dominates the low-momentum part, and the core electron-positron momentum dominates the high momentum part of the momentum distribution. 
For positrons trapped at vacancies the total annihilation rate goes down and the positron lifetime goes up [1]. The partial annihilation rates decrease for both core and valence electron annihilation; however, the decrease in the core electron annihilation rate is much larger than the decrease in the valence electron annihilation rates.

The elemental specificity of the method has been demonstrated in earlier works $[4,5]$. Here we discuss the lattice structure dependence of the Doppler spectrum, using as examples diamond and graphite, $\alpha$ and $\beta$ tin, and amorphous and crystalline $\mathrm{Si}$. Defects in deformed $\mathrm{Cu}$, and vacancy clusters in $\mathrm{Fe}$ are compared with corresponding defect-free or well-annealed samples. It is shown that two different structures of the same element produce significantly different Doppler spectra. The differences are illustrated $[5,6]$ by plotting the ratio curves (with respect to another material) rather than the momentum distribution itself. Our experimental results indicate that the positions of the peaks in the ratio curves depend on the elemental nature of the core electrons, while the amplitude of the peak varies with the lattice structure or the geometry of the defect. The impact of the directional asymmetry on the momentum distribution of the core electrons is being investigated in a separate study [8].

\section{EXPERIMENTAL AND THEORETICAL METHODS}

The experiments were performed using a new coincidence-detector set-up, described elsewhere [5]. The set-up is optimized to record the energies of the two photons when they enter the detector system in time coincidence. The simultaneous detection of both photons allows us to suppress the contributions of the background events that dominate the tail region of the annihilation spectrum of a traditional single detector set-up. The new system attains a peak-to-background ratio of $10^{5}$ (in comparison, the single detector set-up can only attain a ratio of $\approx 200$ ). As a result, we can identify the slope variations originating from annihilations with inner shell electrons.

First-principles calculations have been done to complement the experimental work. The experimental technique can naturally be used alone in a phenomenological manner. However, coupling with calculations that model lattice structures and defect geometries provides descriptions of the annihilation site, and enables us to make a detailed interpretation of the experimental data. Our first-principles calculations use the method described in [6] and [9]. The momentum distribution for each electron level is first calculated, these contributions are then summed up, being weighted with the corresponding partial annihilation rates [9]. These rates are calculated using the method of superimposed atoms [10]. (The effect of the lattice structure on the enhancement of the
Table 1. Lifetimes $\tau$ and annihilation rates $\lambda$ in $\left(\mathrm{ns}^{-1}\right)$ for different electron levels in graphite and diamond

\begin{tabular}{lcc}
\hline & Diamond & Graphite \\
\hline$\tau_{\text {calc }}(\mathrm{ps})$ & 93 & 205 \\
$\tau_{\text {exp }}(\mathrm{ps})$ & $115^{*}$ & $215^{+}$ \\
$\lambda_{1 s}$ & 0.113 & 0.024 \\
$\lambda_{2 s}$ & 5.111 & 2.023 \\
$\lambda_{2 p}$ & 5.558 & 2.838 \\
\hline
\end{tabular}

*From [21].

${ }^{\dagger}$ From [22].

annihilation rate was recently studied by Sormann [11].) The electron density and potential for the positron are calculated by superposing the atomic densities and potentials. The total potential affecting the positron is the sum of the Coulomb potential thus obtained, and a correlation potential calculated using the density functional theory [12] within the generalized gradient approximation for the electron-positron correlation [13]. Since the method is based on using atomistic electron wave functions, the good quantitative agreement between theory and experiment in the case of diamond and graphite may be somewhat fortuitous. This is due to the fact that in both cases the major contribution to the Doppler curves up to the momenta $\simeq 30 m_{0} c$ ( $\simeq 4$ a.u.) is due to the $2 s$ and $2 p$ electrons, responsible for the bond formation, which is not taken properly into account in the atomic superposition calculation. Graphite is highly anisotropic, therefore the use of a directionally averaged positron wave function is also not a good approximation. For $\mathrm{Sn}$, on the other hand, the method is expected to work better, since here the contributions arising from core electrons dominate the behavior of the curve at relatively low momenta. This calculation also gives positron lifetimes that are shown below (Tables 1 and 2) to be in a good agreement with the experiment.

\section{RESULTS AND DISCUSSION}

Fig. 1(a) shows the momentum distributions for diamond and graphite. A 2D-ACAR measurement [14] shows a clear difference in the momentum distribution between the orientations parallel and perpendicular to the crystallographic $c$-axis of graphite. However, the behaviors above $10 m_{0} c(\approx 1.4$ a.u.) are quite similar [14]. The

Table 2. Lifetimes $\tau$ and annihilation rates $\lambda$ (in $\mathrm{ns}^{-1}$ ) for the outermost core electron levels in $\alpha$ and $\beta$ tin

\begin{tabular}{lll}
\hline & $\alpha-\mathrm{Sn}$ & $\beta-\mathrm{Sn}$ \\
\hline$\tau_{\text {calc }}(\mathrm{ps})$ & 286 & 201 \\
$\tau_{\text {exp }}(\mathrm{ps})$ & $289^{*}$ & $201^{\dagger}$ \\
$\lambda_{4 s}$ & 0.009 & 0.021 \\
$\lambda_{4 p}$ & 0.044 & 0.100 \\
$\lambda_{4 d}$ & 0.303 & 0.650 \\
\hline
\end{tabular}

*From [23].

'From [24]. 


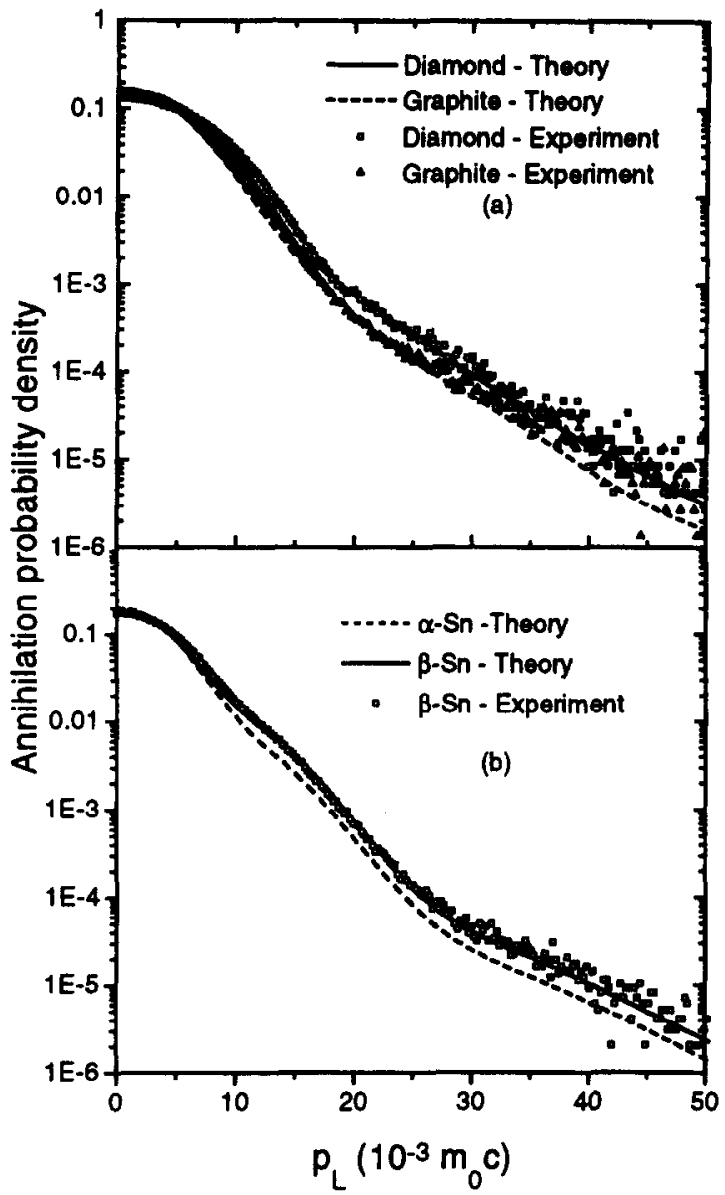

Fig. 1. Positron annihilation probability density for diamond (squares, experiment; solid line, theory) and graphite (triangles, experiment; dashed line, theory). (b) Positron annihilation probability density for $\alpha-S n$ (dashed linc) and $\beta$-Sn (squares, experiment; solid line, theory). 1 a.u. $\simeq 7.3 \times 10^{-3} m_{0} c$.

difference between the momentum distributions for diamond and graphite in our experiments and calculations is seen bettcr by plotting the same curve normalized with respect to another element, in this case $\mathrm{Si}$, because of the availability of high-quality $\mathrm{Si}$ samples (Fig. 2). The normalization procedure is described in [5]. This figure clearly shows that position of the peak is the same for both diamond and graphite, and is indicative of the elemental difference between these materials and $\mathrm{Si}$. It also shows that the amplitude of the peak is different. This difference can be explained in terms of the annihilation rates in both systems. The calculated rates are tabulated in Table 1. In the graphite structure there is plenty of space for the positron wave function between the layers and, therefore, the annihilation rates with the different electron levels are small, resulting in a small total annihilation rate and, therefore, a long lifetime. In contrast, diamond is very closely packed, and the positron is squeezed into the small interstitial space. This results in high annihilation rates and in a very short lifetime (see Table 1).

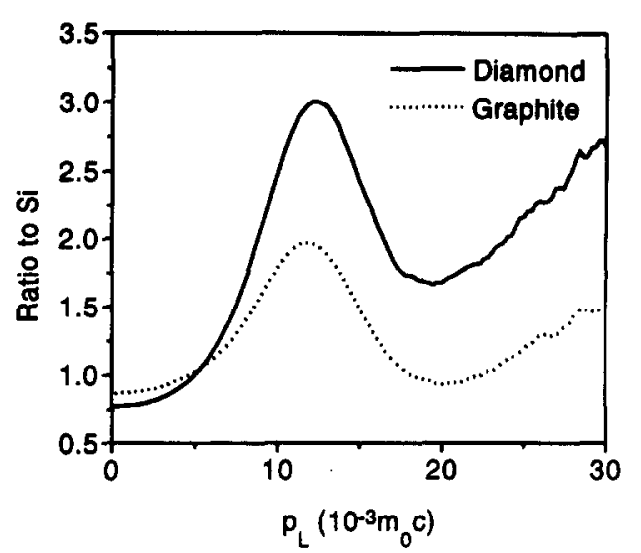

Fig. 2. Experimental positron annihilation probability density for graphite and diamond normalized to Si. $1 \mathrm{a} . \mathrm{u} . \simeq 7.3 \times 10^{-3} m_{0} c$.

Another example of different lattice structures of the same element is tin, which undergoes a phase transition from the diamond structure $\alpha$ phase to the tetragonal $\beta$ phase slightly below room temperature. Fig. 1 (b) clearly shows the difference in the calculated Doppler curves. The core annihilation rates tabulated in Table 2 also show this difference. In $\beta-S n$, the open volume is smaller and the positron wave function has to penetrate deeper into the core region, which increases the annihilation rates with the core electrons, thus broadening the momentum distribution. Since alpha $\mathrm{Sn}$ is difficult to obtain and changes to the beta phase at room temperature, measurements were only done for beta Sn.

Positrons are easily trapped at open volume defects owing to the missing ionic cores. In addition to the relaxation caused by the intrinsic structure of the defect, localized positrons may also alter the atomic coordinates around a defect site $[15,16]$. These two effects will produce similar effects encountered earlier, i.e. they will alter the overlap of the positron with the inner shell electrons. In order to clarify this effect we have studied elements containing different kinds of open volume defects. the ratio curves for defect-free and $\mathrm{N}$ implanted Fe with respect to $\mathrm{Si}$ are shown in Fig. 3(a). The defects in $\mathrm{N}$-implanted $\mathrm{Fe}$ are mostly vacancy clusters, as monovacancies are not stable at room temperature [17]. The figure clearly shows that the peak positions are identical, whereas the amplitudes are quite different. A similar pattern is observed for deformed and annealed $\mathrm{Cu}$ [Fig. 3(b)]. The defects in deformed $\mathrm{Cu}$ are believed to be mainly dislocations and vacancy loops [18].

In the case of amorphous vs crystalline $\mathrm{Si}$, Fig. 3(c), the difference is also clearly visible. Note that even in this case, the amplitude changes almost by a factor of 2 , whereas the corresponding change in the traditional defect parameter $S$ is only the order of a few percentage points. Because of this difference between amorphous and diamond structure, it is expected that this technique 


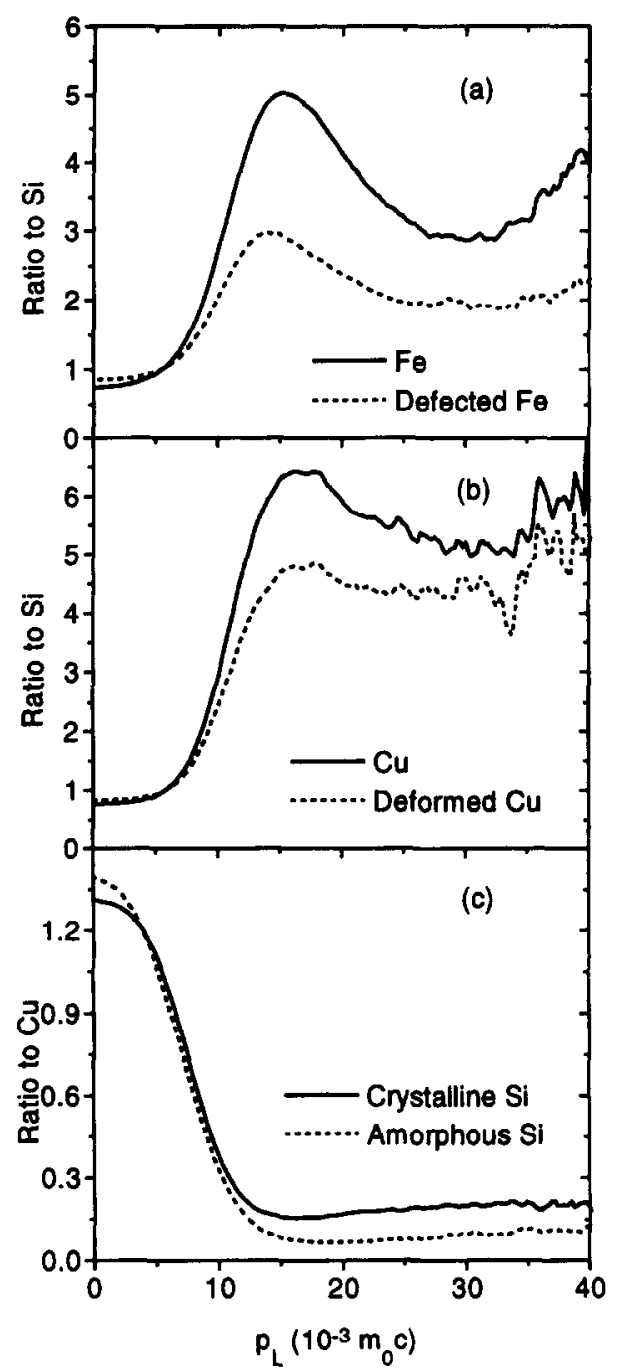

Fig. 3. Experimental positron annihilation probability densities for (a) bulk $\mathrm{Fe}$ and $\mathrm{Fe}$ containing microvoids, (b) bulk and mechanically deformed $\mathrm{Cu}$ (containing dislocations), and (c) crystalline and amorphous Si. The data in (a) and (b) are normalized to $\mathrm{Si}$; the data in (c) is normalized to $\mathrm{Cu}$. 1 a.u. $=7.3 \times 10^{-1} m_{0} c$.

could be used successfully for the characterization of diamond-like features in amorphous carbon films [19].

In the case of both graphite vs diamond and $\alpha$ vs $\beta$ tin, the positron lifetimes are different enough to distinguish easily between the two structures. Positron lifetimes in defect-free materials or the same material containing monovacancies, divacancies, vacancy clusters or dislocations are very different, indicating that the positron lifetime spectroscopy can be used to identify these defects, and it would appear that there is no clear advantage involved in using the momentum distribution measurement. However, positron lifetime spectroscopy is relatively insensitive to changes in the core annihilation rates. The fact that the momentum distribution technique can look at contributions from many electronic levels allows it to identify impurities bound to vacancies, even when the positron lifetime remains unaltered. This has been demonstrated in the case of zinc-impurity phosphorous-vacancy pairs $\left(\mathrm{Zn}-\mathrm{V}_{\mathrm{p}}\right)$ in InP [4].

The good agreement between the theoretical and experimental momentum distributions as well as the calculated and measured positron lifetimes gives credence to the theoretical models for positron states and annihilation characteristics. This supports the conclusion [9] that the overlaps of the positron and core electron densities are well described and that the generalized gradient approximation for electron-positron correlation [13] reproduces faithfully the core annihilation rates for defect-free bulk materials. This is a good starting point for considering the theory for positrons trapped by defects in solids. In that case one has to consider the more complicated effects of the lattice relaxation and the finite positron density $[16,20]$.

\section{CONCLUSION}

We have performed experiments and first-principles calculations which show that the lattice structure of the smaple has a significant effect on the positron-electron annihilation spectrum, even on the core electron part of the momentum distribution. This feature can be utilized for the identification of the environment at the positron annihilation site, yielding information about the chemical composition as well as the lattice structure and lattice relaxations. Since positrons trap selectively at open volume sites prior to annihilation, this is an excellent technique for studying vacancy-impurity complexes in materials.

Acknowledgements-This work was supported in part by U.S. DOE under contract No. DE-AC02-76CH00016.

\section{REFERENCES}

1. For reviews see: Hautojärvi, P. and Corbel, C., in Proceedings of the International School of Physics Enrico Fermi 1993, ed. A. Dupasquier. IOS press, Amsterdam, 1995; Puska, M. J., Phys. Stat. Sol. (a), 1987, 102, 11 and references therein; Puska, M. J. and Nieminen, R. M., Rev. Mod. Phys., 1994, 66, 841.

2. Lynn, K.G., MacDonald, J.R., Boie, R.A., Feldman, L.C., Gabbe, J.D., Robbins, M.F., Bonderup, E. and Golovchenko, S.J., Phys. Rev. Lett., 1977, 38, 241; Lynn, K.G., Dickman, J.E., Brown, W.L., Robbins, M.F. and Bonderup, E., Phys. Rev. B, 1979, 20, 3566.

3. MacDonald, J. R., Lynn. K. G., Boie, R. A. and Robbins, M. F., Nucl. Instr. Meth., 1978, 153, 189.

4. Alatalo, M., Kauppinen, H., Saarinen, K., Puska, M. J., M äkinen, J., Hautojärvi, P. and Nieminen, R. M., Phys. Rev. B, 1995, 51, 4176.

5. Asoka-Kumar, P., Alatalo, M., Ghosh, V. J., Kruseman, A. C., Nielsen, B. and Lynn, K. G., Phys. Rev. Lett., 1996, 77. 2097.

6. Ghosh, V.J., Alatalo, M., Asoka-Kumar, P., Lynn, K.G. and Kruseman, A.C., Appl. Surf. Sci., 1997, 116, 278.

7. Mäkinen, J., Laine, T., Partanen, J., Saarinen, K., Hautojārvi, P., Tappura, K., Hakkarainen, T., Asonen, H., 
Pessa, M., Kauppinen, J.P., Vānttinen, K., Paalanen, M.A. and Likonen, J., Phys. Rev. B, 1996, 53, 7851; Laine, T., Măkinen, J., Saarinen, K., Hautojārvi, P., Corbel, C., Fille, M.L. and Gribart, P., Phys. Rev. B, 1996, 53, 11025 ; Szpala, S., Asoka-Kumar, P., Nielsen, B., Peng, J.P., Hayakawa, S., Lynn, K.G. and Grossman, H.S., Phys. Rev. B, 1996, 54, 4722; Saarinen, K., Laine, T., Skog, K., Mākinen, J., Hautojārvi, P., Rakkenus, K., Uusimaa, P., Salokatue, A. and Pessa, M., Phys. Rev. Lett., 1996, 77, 3407; Laine, T. et al., Phys. Rev. B, in press.

8. Petkov, M. et al., in preparation.

9. Alatalo, M., Barbiellini, B., Hakala, M., Kauppinen, H., Korhonen, T., Puska, M. J., Saarincn, K., Hautojärvi, P. and Nieminen, R. M., Phys. Rev. B, 1996, 54, 2397.

10. Puska, M. J. and Nieminen, R. M., J. Phys. F, 1983, 13, 2695 ,

11. Sormann, H., Phys. Rev. B, 1996, 54, 4558.

12. Boroński, E. and Nieminen, R.M., Phys. Rev. B, 1986, 34, 3280; Arponen, J. and Pajanne, E., Ann. Phys. (N.Y.), 1979, 121, 343.

13. Barbiellini, B., Puska, M.J., Torsti, T. and Nieminen, R.M., Phys. Rev. B, 1995, 51, 7341; Barbiellini, B., Puska, M. J., Korhonen, T., Harju, A., Torsti, T. and Nieminen, R. M., Phys. Rev. B, 1996, 53, 16201.
14. Cartier, E., Heinrich, F., Pfluger, P. and Güntherod, J.-J., Solid State Comm., 1981, 38, 985.

15. Laasonen, K., Alatalo, M., Puska, M.J. and Nieminen, R.M., J. Phys.: Condens. Matter, 1991, 3, 7217; Gilgien, L., Galli, G., Gygi, F. and Car, R., Phys. Rev. Lett., 1994, 72, 3214.

16. Puska, M. J., Seitsonen, A. P. and Nieminen, R. M., Phys. Rev. B, 1995, 52, 10947.

17. Vehanen, A., Hautojärvi, P., Johansson, J., Yli-Kauppila, J. and Moser, P., Phys. Rev. B, 1982, 25, 762.

18. Dauwe, C., Dorikens, M., Dorikens-Vanpraet, L. and Segers, D., Appl. Phys., 1974, 5, 117.

19. Poutch, J. and Altcrovitz, S.A., cd., Properties and Preparation of Amorphous Carbon Films. Trans. Tech. Publications, Aedermannsdorf, Switzerland, 1989.

20. Korhonen, T., Puska, M. J. and Nieminen, R. M., Phys. Rev. $B, 1996,54,15016$.

21. Dannefaer, S., J. Phys. C., 1982, 15, 599.

22. Shimotomai, N., Takahashi, T., Doyama, M. and Iwata, T., in Positron Annihilation, ed. P. G. Coleman et al. North Holland, Amsterdam, 1982, p. 483.

23. Puff, W. and Mascher, P., J. Phys. F, 1984, 14, L231.

24. Puff, W., unpublished. 